\title{
An oncolytic adenovirus that expresses the HAb18 and interleukin 24 genes exhibits enhanced antitumor activity in hepatocellular carcinoma cells
}

\author{
Sujing Yuan ${ }^{1}$, Xianlong Fang ${ }^{1}$, Yanni Xu ${ }^{2}$, Aimin Ni ${ }^{1}$, Xin-Yuan Liu ${ }^{1}$, Liang Chu ${ }^{1,3}$ \\ ${ }^{1}$ State Key Laboratory of Cell Biology, Institute of Biochemistry and Cell Biology, Shanghai Institutes for Biological Sciences, \\ Chinese Academy of Sciences, Shanghai 200031, P. R. China \\ ${ }^{2}$ College of Life Sciences, Northwest Agriculture and Forestry University, Yangling 712100, P. R. China \\ ${ }^{3}$ Jiangsu Center for the Collaboration and Innovation of Cancer Biotherapy, Cancer Institute, Xuzhou Medical College, Xuzhou \\ 221002, P. R. China \\ Correspondence to: Xin-Yuan Liu, email: xyliu@sibcb.ac.cn \\ Liang Chu, email: Ichu@sibcb.ac.cn \\ Keywords: oncolytic adenovirus, HAb 18, CD 147, interleukin 24, hepatocellular carcinoma \\ Received: January 18,2016 Accepted: July 26, $2016 \quad$ Published: August 09, 2016
}

\section{ABSTRACT}

\begin{abstract}
Hepatocellular carcinoma (HCC) is characterized by alterations in multiple genes. High expression of CD147 on the surface of HCC cells promotes proliferation. The monoclonal antibody HAb18 recognizes CD147. We constructed an oncolytic adenoviral vector to express HAb18 (ZD55-HAb18) in HCC cells. Interleukin 24 (IL24) was co-expressed through the use of an F2A linker. ZD55-HAb18-IL24 decreased HCC cell viability to a greater degree than either ZD55-HAb18 or ZD55-IL24 alone. ZD55-HAb18-IL24 also induced apoptosis and autophagy in PLC/PRF/5 HCC cells. Intratumoral injection of ZD55-HAb18-IL24 repressed tumor growth in a PLC/PRF/5 xenograft model. Our results suggest that antibody-antitumor gene conjugation elicited a stronger antitumor effect than the antibody alone, and that this strategy could broaden the applications of antibody-based therapies in HCC.
\end{abstract}

\section{INTRODUCTION}

Hepatocellular carcinoma (HCC) is a multi-factorial disease that involves cross-talk between several pathways [1]. Combination approaches for HCC therapy based on multi-targeted conjugates have significant advantages [2]. Combination endostatin/sFlt-1 antiangiogenic gene therapy was shown to be highly effective in a rat model of HCC [3]. Complete eradication of HCC was achieved by combined vasostatin gene therapy and $\mathrm{B} 7 \mathrm{H} 3$-mediated immunotherapy [4]. Drozdzik et al. demonstrated that a suicide gene in combination with interleukin-12 was more efficient than therapy with one gene alone in a murine model of HCC [5].

CD147 is a transmembrane glycoprotein that belongs to the immunoglobulin superfamily and is highly enriched on the surface of human HCC cells [6]. CD147 plays a critical role in tumor progression and metastasis [7]. Recent studies have revealed that knockdown of CD147 reduced cell proliferation and improved chemo- sensitivity in many cancer cells $[8,9]$. HAb18 is a singlechain monoclonal antibody fragment (scFv). The variable regions of the heavy chain and light chains are linked by a (GGGS) ${ }_{3}$ peptide. HAb18 is secreted by hybridoma cells in BALB/c mice immunized with human HCC tissue extracts. The antigen recognized by HAb18 is CD147, which was identified in a screen of an HCC cDNA library [10-12]. Xu et al. reported that blocking CD147 with $\mathrm{HAb} 18 \mathrm{mAb}$ inhibited $\mathrm{HCC}$ growth and metastasis in vivo [13].

Interleukin 24 (IL24) is a cytokine that belongs to the IL-10 family of cytokines [14]. Preclinical studies have shown that ectopic expression of IL24 induces apoptosis in cancer cells with no significant cytotoxicity to normal cells $[15,16]$. IL24 has synergistic effects in various human cancers when combined with other agents. For example, Ad-IL24 combined with a selective inhibitor of EGFR (gefitnib) induces apoptotic cell death in nonsmall cell lung cancer [17]. In another study, treatment of Her-2/neu-overexpressing breast cancer cells with AdIL24 in combination with a monoclonal antibody targeting 
the Her-2/neu receptor inhibited cell growth [18]. These studies indicate therapeutic antibodies combined with IL24 might enhance antitumor efficacy.

Oncolytic adenoviral vectors are promising cancer therapies [19-21]. The ONYX-015 vector was engineered to lack expression of the E1B55KD viral protein. Reduced replication of ONYX-015 in normal cells results from defective export of late viral RNA. This is because the E1B55KD protein facilitates preferential transport of viral RNA during the late stages of oncolytic adenoviral infection $[22,23]$. We constructed the oncolytic adenovirus ZD55, in which the E1B55KD gene was deleted and a cloning site added in order to insert foreign antitumor genes [24]. Previous studies have shown that ZD55 carrying the IL24 gene could selectively replicate in tumor cells and inhibit cell growth more effectively than ONYX015 and a replication-defective adenovirus carrying the IL24 gene [25]. In another study, a combination of ZD55 carrying the TRAIL gene and ZD55 carrying the Smac gene suppressed the growth of HCC tumors in mice [26].

In this study, we used ZD55 to express HAb18IL24 linked by a foot-and-mouth-disease virus (FMDV)derived 2A self-processing peptide (F2A). ZD55-HAb18IL24 decreased HCC cell viability, induced apoptosis and autophagy, and inhibited tumor growth in a PLC/ PRF/5 xenograft model. Additionally, ZD55-HAb18IL24 displayed potent antiangiogenic activity in vivo. Our results indicate oncolytic adenoviruses carrying a combination of a therapeutic monoclonal antibody and a cytokine may be effective HCC therapies.

\section{RESULTS}

\section{CD147 is highly expressed in HCC and promotes cell proliferation and chemoresistance}

CD147 expression in HCC tissue (T) and adjacent non-cancerous tissue $(\mathrm{N})$ was evaluated by quantitative real-time PCR (qRT-PCR) (Figure 1A). CD147 was found to be overexpressed in tumor compared to normal tissue. Examination of CD147 expression in several HCC cell lines revealed higher CD147 mRNA and protein expression in cancer cells compared to normal human hepatocytes (QSG-7701 cells) (Figure 1B and 1C).

To investigate the effects of CD147 on HCC cell proliferation, we overexpressed CD147 in PLC/PRF/5, Hep3B, and HepG2 cells using a lentiviral infection strategy. Cells that stably overexpressed CD147 were selected with puromycin (Figure 1D and 1E). Overexpression of CD147 promoted cell proliferation (Figure 1F) and conferred resistance to cytotoxic chemotherapy (Figure 1G). We next knocked down CD147 using lentivirus-mediated shRNA (Figure 1H and 1I). Knockdown of CD147 inhibited cell growth (Figure 1J) and sensitized the cells to chemotherapy (Figure $1 \mathrm{~K})$. These results demonstrated that CD147 enhanced cell proliferation and protected cells from the effects of chemotherapy, suggesting that it might be an attractive therapeutic target in HCC.

\section{ZD55-HAb18-IL24 exhibits enhanced cytotoxicity in vitro}

The effects of CD147 prompted us to assess whether inhibition of CD147 with the monoclonal antibody HAb18 could have therapeutic efficacy. We also simultaneously introduced IL24 into the oncolytic adenoviral vector since multi-target agents have been widely investigated for cancer treatment. We hypothesized that a combination of HAb18 and IL24 could improve the therapeutic efficacy. To test this hypothesis, we constructed an oncolytic adenovirus that expressed the HAb18 (ZD55-HAb18) and IL24 (ZD55-IL24) genes. We linked the two genes with an F2A sequence and cloned the product into an oncolytic adenoviral vector to generate ZD55-HAb18-IL24 (Figure 2A). PCR analysis of the E1B-55KD gene confirmed that all the oncolytic adenoviruses were free of wild-type adenovirus contamination (Figure 2B).

The expression of exogenous HAb18, IL24, and GFP in PLC/PRF/5 cells was detected by western blot (Figure 2C). PLC/PRF/5 cells infected with ZD55HAb18-IL24 expressed HAb18 and IL24 individually with bands corresponding to the correct molecular weights. The expression of both proteins in ZD55-HAb18-IL24-treated cells was similar to expression in cells infected with either ZD55-HAb18 or ZD55-IL24 individually, indicating that the F2A peptides mediated efficient generation of the individual HAb18 and IL24 proteins. We observed decreases in MMP-2 and MMP-9 expression in ZD55HAb18- and ZD55-HAb18-IL24-treated PLC/PRF/5 cells, which suggested that oncolytic adenoviral expression of mAb HAb18 inhibited CD147 function (Figure 2D).

To compare the abilities of the oncolytic adenoviruses to replicate in tumor and normal cell lines, $\mathrm{PLC} / \mathrm{PRF} / 5$ and QSG-7701 cells were infected with oncolytic adenoviruses at an MOI of 10 . The relative replication efficacy of the oncolytic adenoviruses was measured by qRT-PCR of adenovirus gene E1A cDNA. The oncolytic adenoviruses replicated effectively in tumor cells (Figure 2E). In contrast, the replication ability was significantly reduced in normal cells. These results demonstrated that oncolytic adenoviruses selectively replicated in tumor cells.

We next evaluated the cytotoxicity of ZD55$\mathrm{HAb} 18-\mathrm{IL} 24$. PLC/PRF/5 HCC cells were infected with ZD55-HAb18-IL24 at a series of MOIs from 0.05-20. Cell viability was measured 4 days later and the IC50 of ZD55-HAb18-IL24 determined. ZD55-HAb18-IL24 significantly inhibited PLC/PRF/5 cell growth (IC50 = $9.21 \pm 1.24 \mathrm{MOI})$. Importantly, a rare cytopathic effect of ZD55-HAb18-IL24 was observed in QSG-7701 cells (even at an MOI of 20), suggesting that ZD55-HAb18- 
A

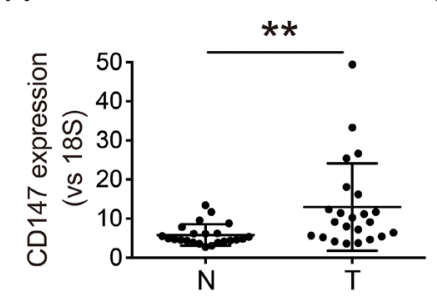

D

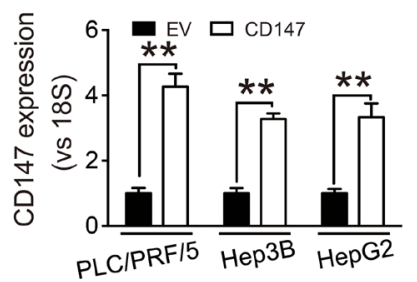

G

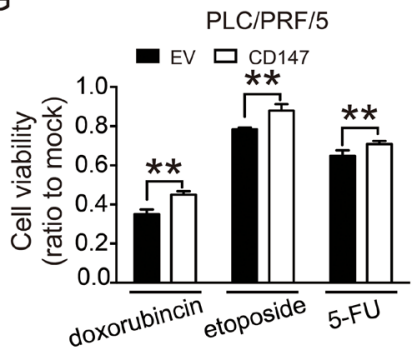

H

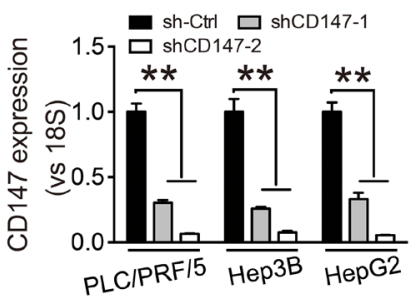

K
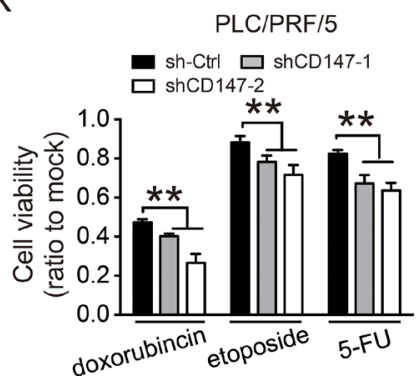

B

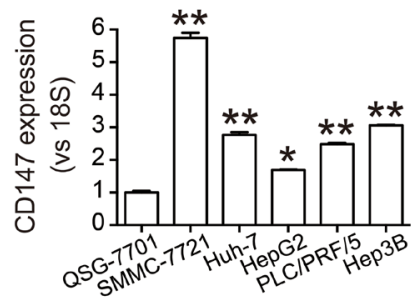

E

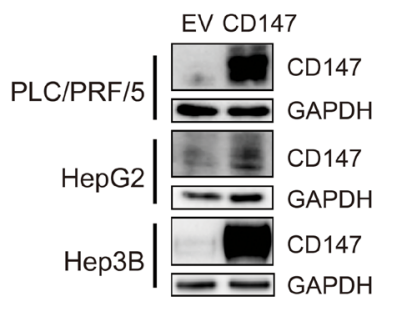

F

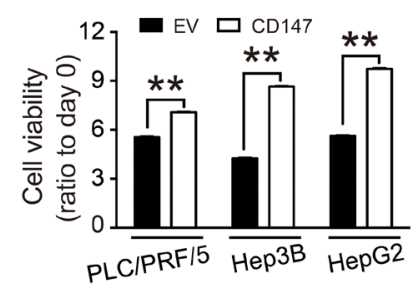

Нер3в

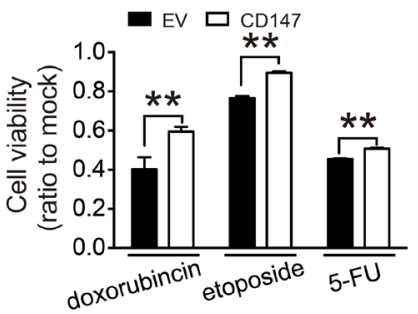

I

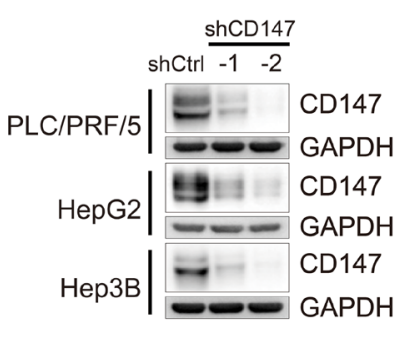

J
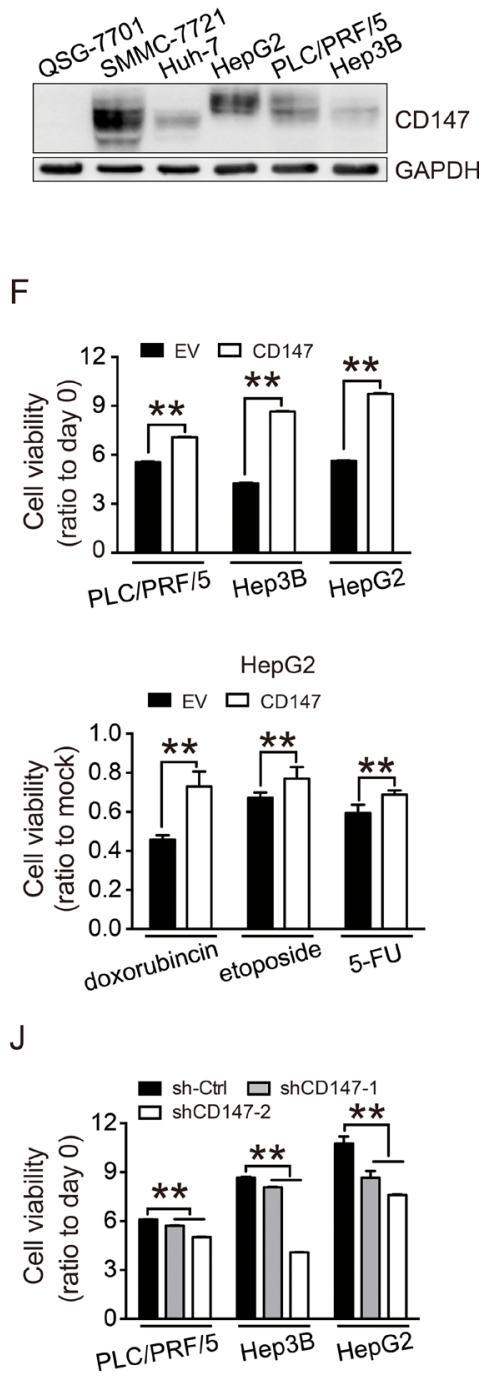

F

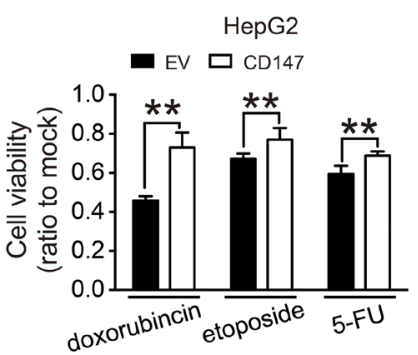

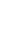

CD147

GAPDH

Hep3B

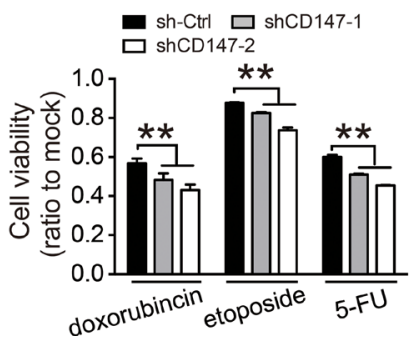

Figure 1: CD147 is highly expressed in HCC and promotes cell proliferation and chemoresistance. A. Levels of CD147 mRNA in 23 HCC tissue (T) and adjacent non-cancerous tissue $(\mathrm{N})$ were measured by qRT-PCR. B, C. Expression of CD147 in HCC cell lines (SMMC-7721, Huh-7, HepG2, PLC/PRF/5, and Hep3B) and normal hepatocytes (QSG-7701) was measured by qRT-PCR (B) and western blot (C). D, E. CD147 overexpression was confirmed by qRT-PCR (D) and western blot (E). F. CD147 overexpression increases proliferation in PLC/PRF/5, Hep3B, and HepG2 cells. G. CD147 overexpression increases the resistance of PLC/PRF/5, Hep3B, and HepG2 cells to cytotoxic chemotherapy. H, I. CD147 knockdown was confirmed by qRT-PCR (H) and western blot (I). J, K. Knockdown of CD147 inhibits cell proliferation (J) and decreases resistance to cytotoxic chemotherapy (K). Cell proliferation was measured using CCK-8 assays at $96(\mathrm{~F}, \mathrm{~J})$ and 48 hours $(\mathrm{G}, \mathrm{K})$. Cells were treated with doxorubicin $(1 \mu \mathrm{g} / \mathrm{mL})$, etoposide $(10 \mu \mathrm{g} / \mathrm{mL})$, and fluorouracil (5-FU; 100 $\mu \mathrm{g} / \mathrm{mL}$ ) for 2 days. Relative cell viability is shown as the fold change compared to mock control cells (G, K). GAPDH was used as a loading control in (C), (E), and (I). The qRT-PCR data in (A), (B), (D), and (H) were normalized to 18S RNA and are shown as the fold change relative to QSG-7701 cells in (B), EV groups in (D), and sh-Ctrl groups in (H). EV, empty vector. Sh-Ctrl, scramble shRNA vector control. All experiments were repeated three times. The bars represent the mean \pm S.D. $(n=3),{ }^{*} \mathrm{p}<0.05,{ }^{* *} \mathrm{p}<0.01$ 

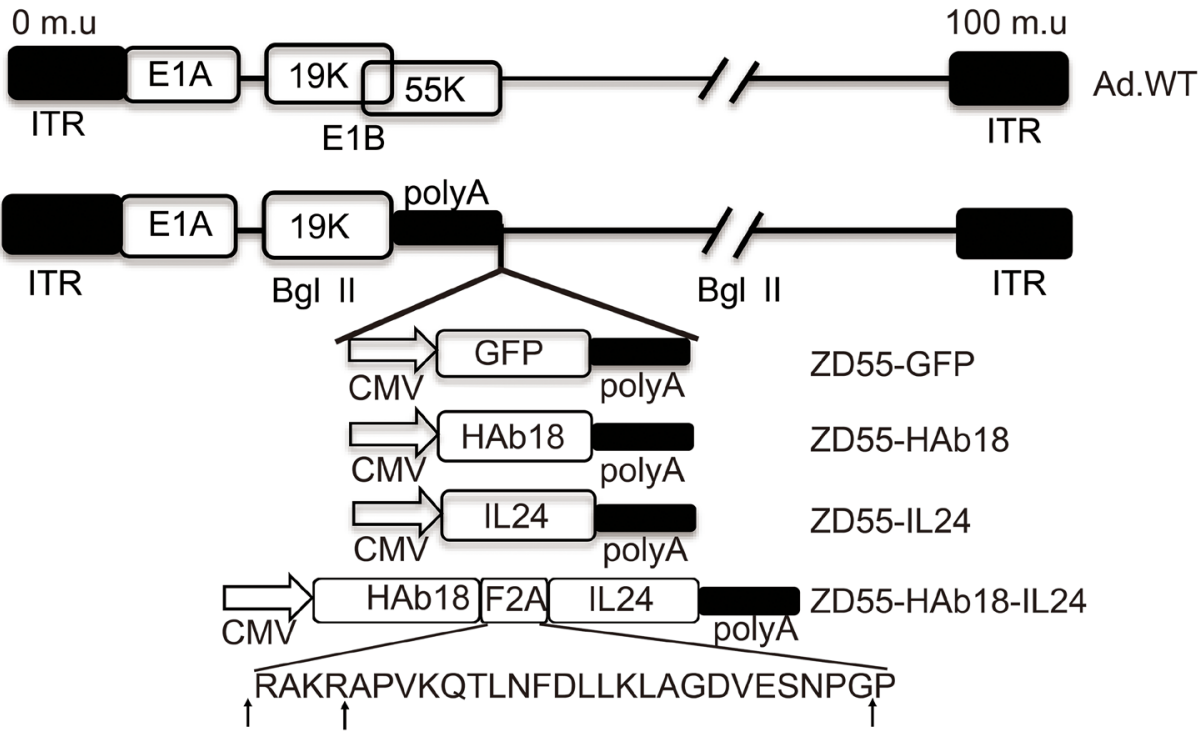

B

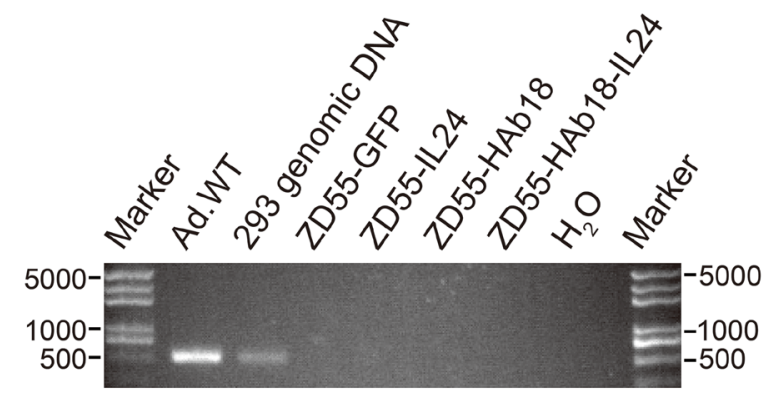

C

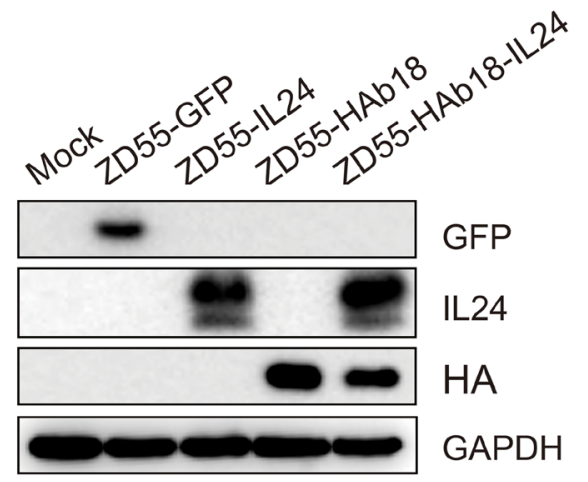

D

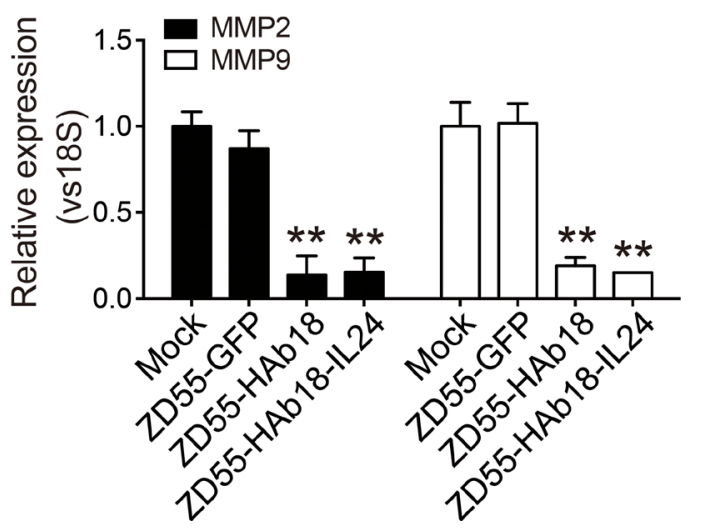

E

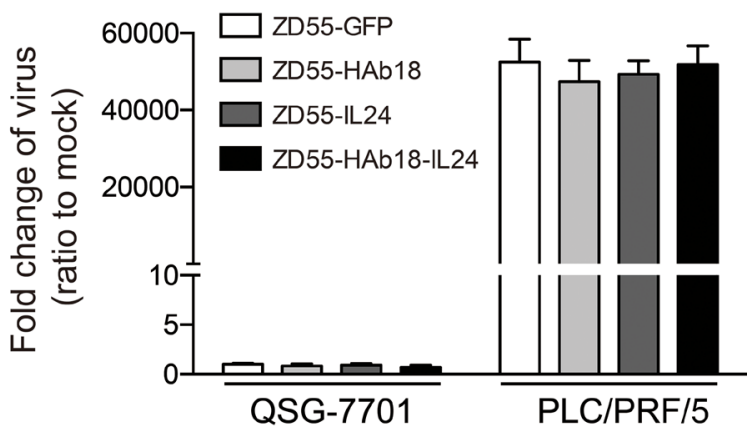

Figure 2: Construction and characterization of the oncolytic adenoviruses. A. Schematic of the structures of the oncolytic adenoviruses. The amino acid sequence of F2A is shown. Arrows indicate the cleavage sites in F2A. Ad.WT, wild-type adenovirus. ITR, inverted terminal repeat. B. Characterization of oncolytic adenoviruses by PCR using the E1B-55KD forward and reverse primers (Supplementary Table S1). All oncolytic adenovirus showed no wild-type contamination. C. Western blot detection of genes carried by ZD55 in PLC/PRF/5 cells 48 hours after oncolytic adenovirus infection (10 MOI). GAPDH was used as a loading control. D. Analysis of MMP-2 and MMP-9 in PLC/PRF/5 cells treated with the indicated oncolytic adenoviruses (MOI of 10) for 48 hours by qRT-PCR. E. Analysis of the replication ability of the oncolytic adenoviruses in normal hepatocytes (QSG-7701) and tumor cells (PLC/PRF/5) by qRTPCR. The adenovirus E1A region was amplified to evaluate viral replication 48 hours after infection (MOI of 10). The qRT-PCR data in (D) and (E) were normalized to $18 \mathrm{~S}$, and are shown as the fold change relative to mock cells. All experiments were repeated three times. The bars represented the mean \pm S.D. $(n=3) .{ }^{*} \mathrm{p}<0.01$. 
IL24 was cytotoxic to cancer cells but not normal cells (Figure 3A). The oncolytic adenoviruses had no effect on the growth of QSG-7701 cells transduced with CD147expressing lentiviruses (Supplementary Figure S1). We next compared the antitumor effects of ZD55-HAb18IL24, ZD55-HAb18, and ZD55-IL24. Cells were infected with the indicated oncolytic adenoviruses at an MOI of 10 for 4 days. ZD55-HAb18-IL24 inhibited HCC cell growth to a greater extent than either ZD55-HAb18 or ZD55-IL24 alone (Figure 3B-3F), indicating ZD55-HAb18-IL24 had higher antitumor efficacy.

A

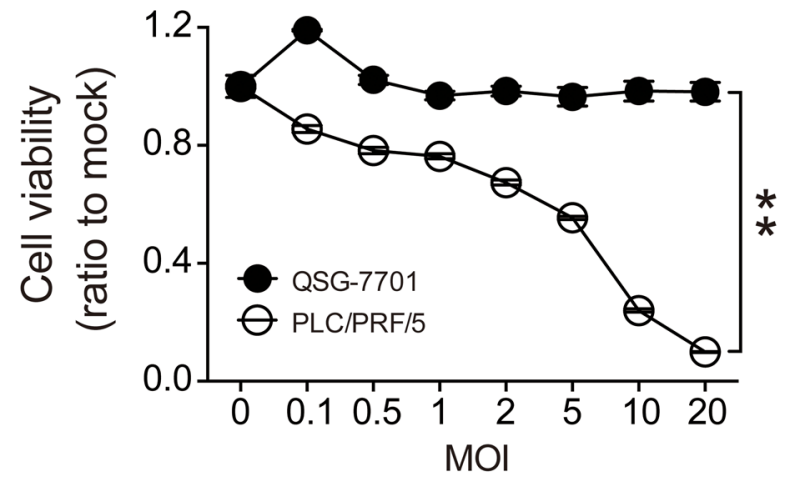

C

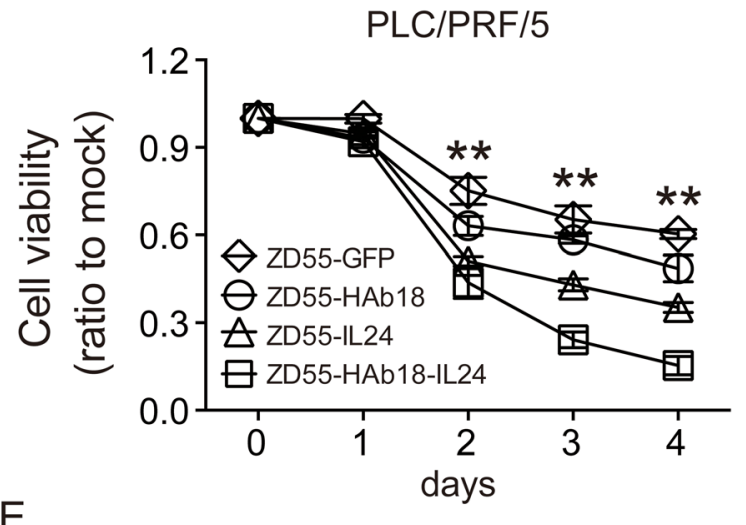

$E$

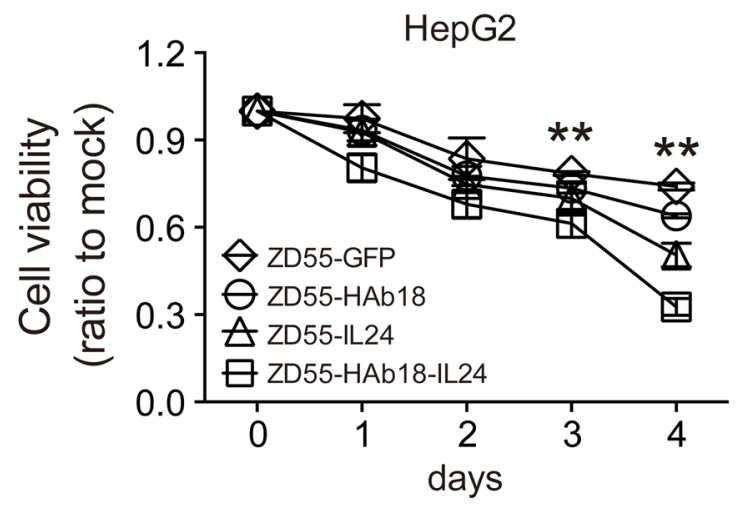

\section{ZD55-HAb18-IL24 induces apoptosis and autophagy}

We assessed whether ZD55-HAb18-IL24 could induce apoptosis in PLC/PRF/5 cells 48 hours after oncolytic adenovirus infection using Annexin V/propidium iodide (PI) staining. The percentage of apoptotic cells (Annexin $\mathrm{V}^{+} / \mathrm{PI}^{+}$) in the ZD55-HAb18-IL24-treated group was higher than in the other groups (Figure 4A). We also detected several key members of caspase-dependent apoptotic signaling cascades by western blot. Enhanced

B

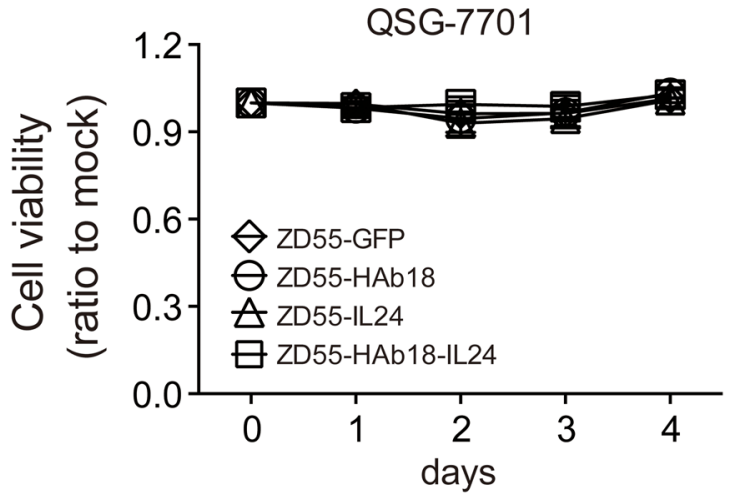

D

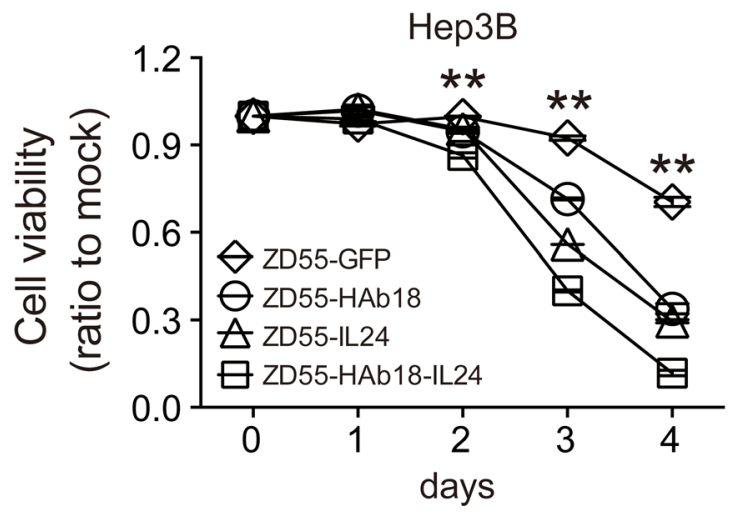

F

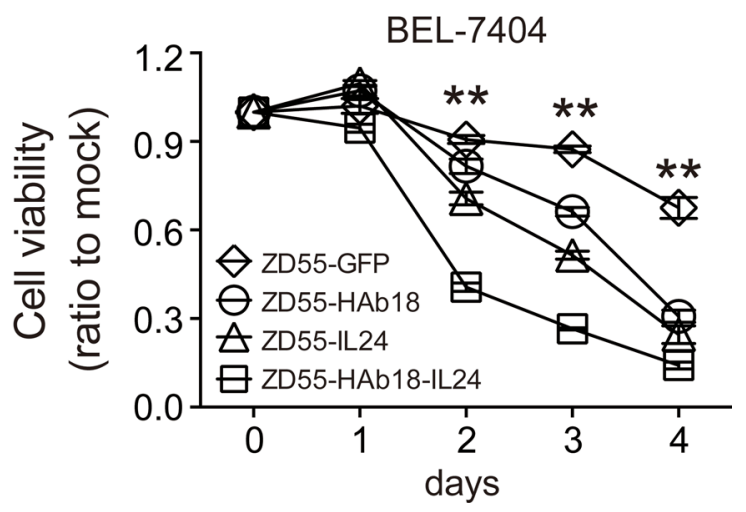

Figure 3: ZD55-HAb18-IL24 displays antitumor activity in vitro. A. The viability of PLC/PRF/5 and QSG-7701 cells infected with ZD55-HAb18-IL24 at the indicated MOIs on day 4. B-F. The viability of QSG-7701, PLC/PRF/5, Hep3B, HepG2, and BEL-7404 cells infected with the indicated oncolytic adenoviruses for 4 days at an MOI of 10. Cell viability was assessed using CCK-8 assays. All experiments were repeated three times. The bars represent the mean \pm S.D. $(n=3),{ }^{*} \mathrm{p}<0.05,{ }^{* *} \mathrm{p}<0.01$. 
A

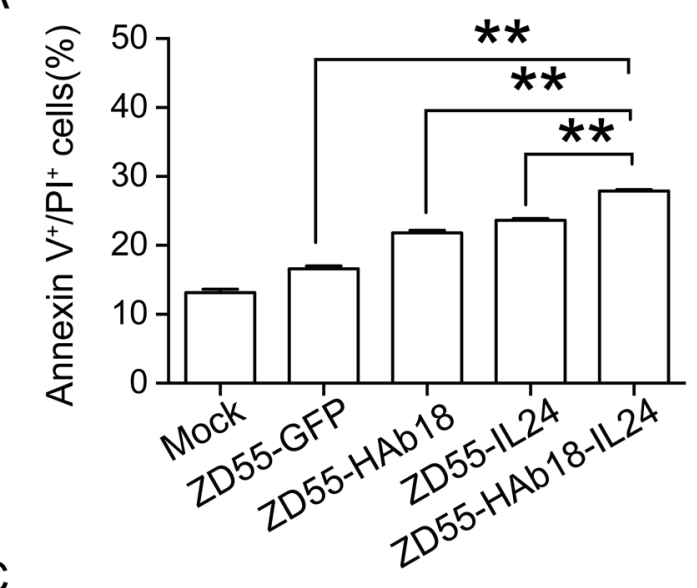

C
B

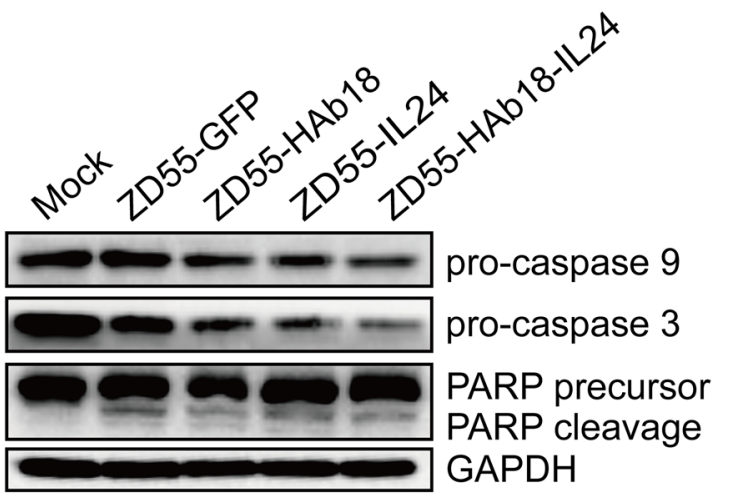

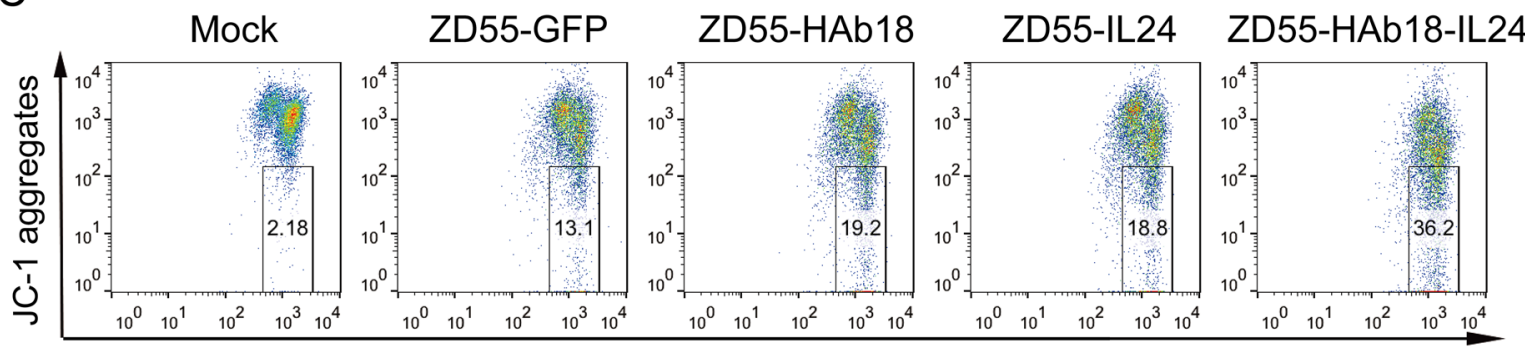

D JC-1 monomers
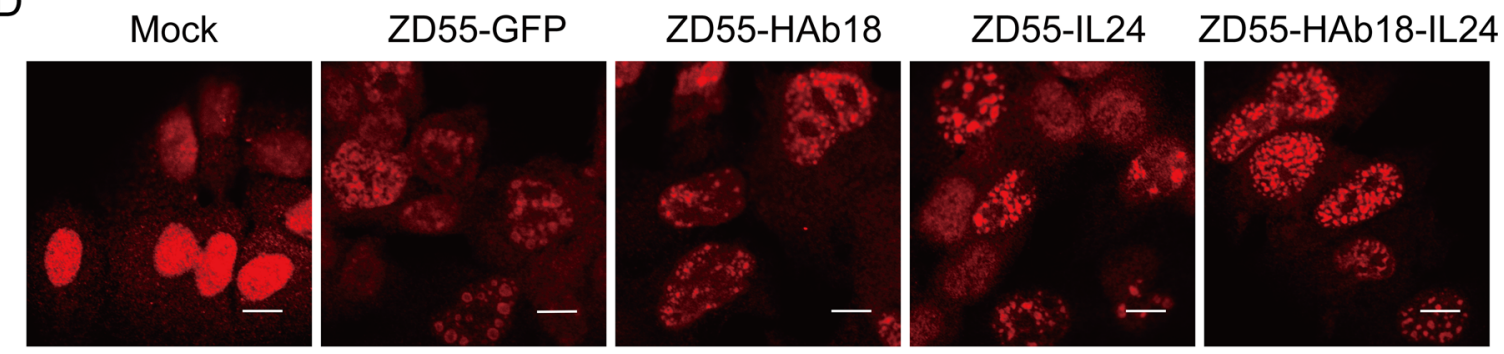

E

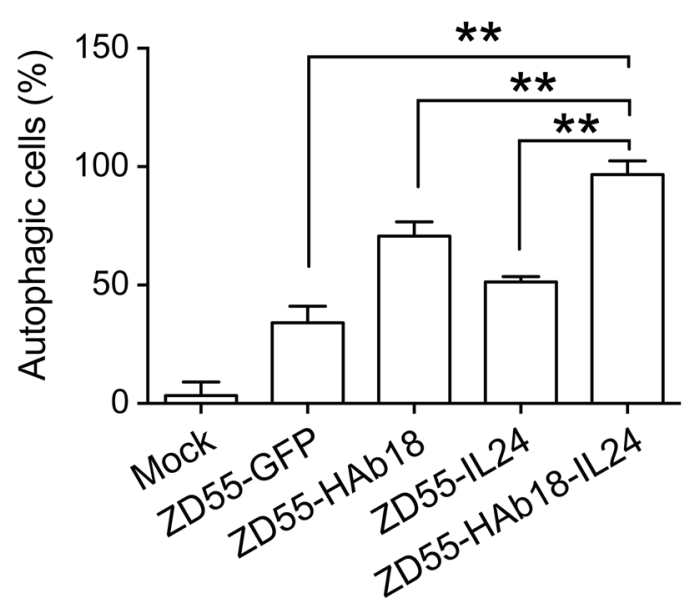

F

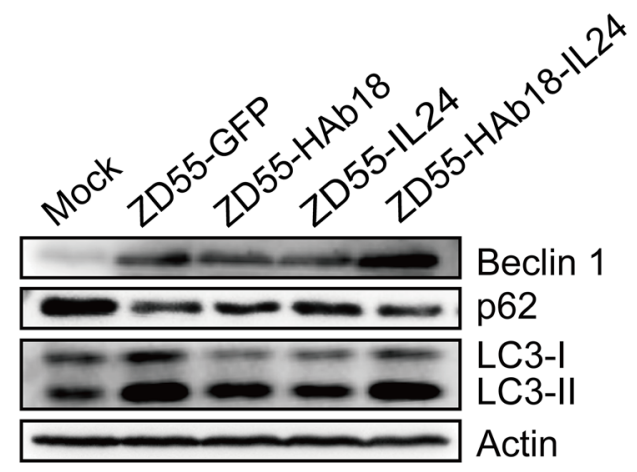

Figure 4: ZD55-HAb18-IL24 induces apoptosis and autophagy in PLC/PRF/5 cells. A. Apoptosis was evaluated in oncolytic adenovirus-infected PLC/PRF/5 cells using Annexin V/PI staining. B. Western blot detection of procaspase-9, procaspase-3, and PARP in $\mathrm{PLC} / \mathrm{PRF} / 5$ cells. GAPDH was used as a loading control. C. Oncolytic adenoviruses alter the mitochondrial membrane potential $(\Delta \psi \mathrm{m})$ in PLC/PRF/5 cells. The numbers in the quadrilateral region show the ratio of cells with altered $\Delta \psi \mathrm{m}$. D. Representative images of LC3 puncta in PLC/PRF/5 cells infected with indicated oncolytic adenoviruses. Scale bars, $10 \mu \mathrm{m}$. E. Statistical data for the three repeats in (D). F. Western blot detection of beclin-1, p62, and LC3-I/II in oncolytic adenovirus-infected PLC/PRF/5 cells. Actin was used as a loading control. PLC/PRF/5 cells were infected with the indicated oncolytic adenoviruses (MOI of 10) for 2 days. All experiments were repeated three times. The bars represent the mean \pm S.D. $(n=3) .{ }^{*} \mathrm{p}<0.01$. 
poly ADP-ribose polymerase (PARP) cleavage, and decreased expression of procaspase- 3 and procaspase- 9 were observed in ZD55-HAb18-IL24-treated PLC/ $\mathrm{PRF} / 5$ cells (Figure 4B). Because cleaved caspase-9 is an essential initiator of the mitochondrial apoptosis signaling pathway, we analyzed changes in the mitochondrial membrane potential $(\Delta \psi \mathrm{m})$ using $\mathrm{JC}-1$ staining. An increase in the number of cells with mitochondrial membrane potential loss was observed in ZD55-HAb18IL24-treated PLC/PRF/5 cells (Figure 4C). These data suggested that ZD55-HAb18-IL24 induced apoptosis in PLC/PRF/5 cells through the mitochondrial pathway.

Previous data indicated CD147 could inhibit autophagy in cancer cells $[27,28]$. Microtubule-associated protein light chain 3 (LC3) is essential for autophagosome formation and is an accurate autophagosome marker. ZD55-HAb18-IL24-treated PLC/PRF/5 cells exhibited a higher percentage of cells with LC3 puncta compared to the other oncolytic adenovirus-treated cells (Figure 4D and 4E). Increased expression of beclin-1 (an autophagy protein), degradation of p62 (an autophagy substrate), and conversion of the nonlipidated form of LC3 (LC3-I) to the phosphatidylethanolamine-conjugated form (LC3-II) were observed in ZD55-HAb18-IL24-treated cells (Figure 4F). These data demonstrated that ZD55-HAb18-IL24 induced autophagy in PLC/PRF/5 cells.

\section{ZD55-HAb18-IL24 suppresses tumor growth in vivo}

To evaluate the therapeutic potential of oncolytic adenoviruses in vivo, oncolytic adenoviruses and corresponding volumes of PBS were intratumorally injected in PLC/PRF/5 xenografts. Treatment with ZD55-HAb18-IL24 delayed tumor growth compared to treatment with the other oncolytic adenoviruses (Figure 5A). The mice were sacrificed 33 days after injection. The tumor volume and weight in the ZD55-HAb18IL24-treated group were $297.8 \pm 67.5 \mathrm{~mm}^{3}$ and $47 \pm$ $0.05 \mathrm{~g}$, respectively. These tumors were significantly smaller than the tumors in the other groups (Figure 5B and 5C), indicating that ZD55-HAb18-IL24 was able to efficiently inhibit PLC/PRF/5 xenograft growth. HAb18 and IL24 expression in tumors was assessed by qRTPCR, and the serum HAb18 level examined by enzymelinked immunosorbent assays (ELISA) at the end of the experiment. ZD55-HAb18-IL24 expressed high levels of HAb18 and IL24 in tumors (Figure 5D). The expression of HAb18 in serum remained relatively high for $>1$ month (Figure 5E). We analyzed the kinetics of HAb18 expression in nude mice bearing $\mathrm{PLC} / \mathrm{PRF} / 5$ tumors. These results demonstrated that ZD55-HAb18 and ZD55HAb18-IL24 efficiently expressed HAb18 (Supplementary Figure S2). Additionally, no significant increases in the expression of aspartate aminotransferase (AST), alanine aminotransferase (ALT), blood urea nitrogen (BUN), or creatinine were observed after treatment (Supplementary Figure S3).

The apoptotic and autophagic effects of ZD55$\mathrm{HAb} 18-\mathrm{IL} 24$ in PLC/PRF/5 xenografts were analyzed using terminal deoxynucleotidyl transferase dUTP nick end labeling (TUNEL) assays. A higher percentage of positive cells was observed in the ZD55-HAb18-IL24treated tumors than in the other groups (Figure $5 \mathrm{~F}$ and 5G). Tumors in the ZD55-HAb18-IL24-treated groups displayed more autophagic structures than tumors in the other groups, and no autophagic structures were observed in PBS-treated tumors by electron microscopy (Figure 5F). Because angiogenesis plays a critical role in tumor progression, we examined the expression of VEGFA in adenovirus-treated cells in vitro and in vivo. A significant decrease in VEGFA expression was observed after adenovirus infection (Supplementary Figure S4). Immunohistochemical staining of CD31, a vascular endothelial marker, revealed reduced microvessel density in ZD55-HAb18-IL24-treated tumors (Figure 5F and 5H).

\section{DISCUSSION}

HCC can result from both genetic and epigenetic alterations [29]. Many factors involved in HCC development are potential therapeutic targets. CD147 is an $\mathrm{HCC}$-associated antigen and plays important roles in $\mathrm{HCC}$ progression [30]. Our data indicate that CD147 promotes HCC cell proliferation and chemoresistance (Figure 1). Overexpression of CD147 was also shown to promote proliferation and chemoresistance in bladder cancer T24 cells (Supplementary Figure S5). These findings suggest that CD147 might be a broad-spectrum target for cancer therapy.

Monoclonal antibodies are advantageous for the treatment of cancer [31]. In order to achieve clinical therapeutic effects, antibody levels in serum must be high [32]. Adeno-associated virus vectors that transferred the anti-HIV mAb gene yielded low levels of antibody [33]. Recombinant oncolytic Newcastle Disease Virus carrying the full-length mouse-human chimeric HAb18 gene (cHAb18) induced HCC cell necrosis and prolonged survival in mice through inhibition of local tumor metastasis [34]. Here, we used the oncolytic adenoviral vector ZD55 to mediate HAb18 mAb expression in combination with the therapeutic cytokine IL24, which were linked by an F2A sequence. High expression of HAb18 and IL24 could still be observed 33 days after injection of ZD55-HAb18-IL24 into nude mice bearing $\mathrm{PLC} / \mathrm{PRF} / 5$ xenograft tumors (Figure 5D and 5E). However, when the vector was injected into to C57/BL6 mice bearing syngeneic colon tumors, low serum HAb18 expression was observed. This could have resulted from defective replication of the oncolytic adenovirus in murine cells (Supplementary Figure S6). 
A

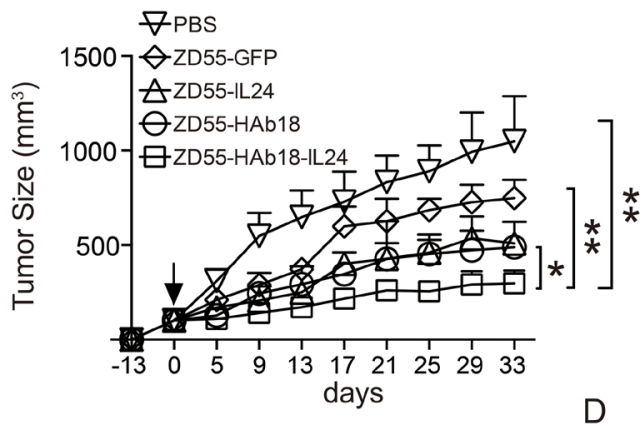

C

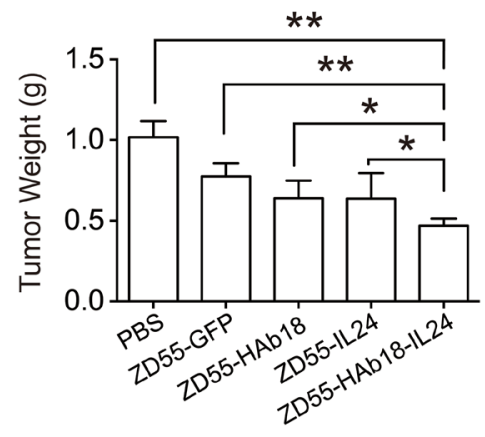

E

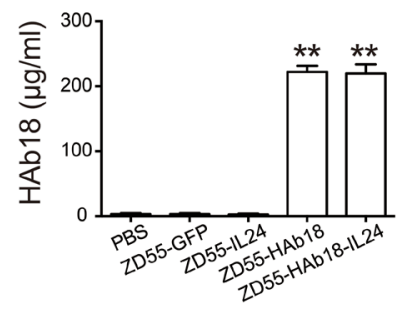

G

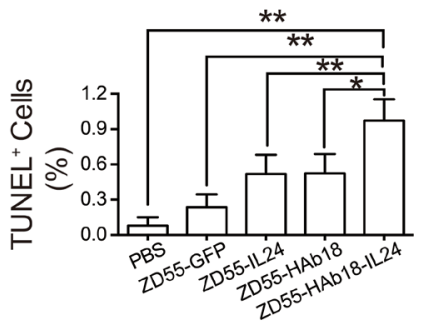

$\mathrm{H}$

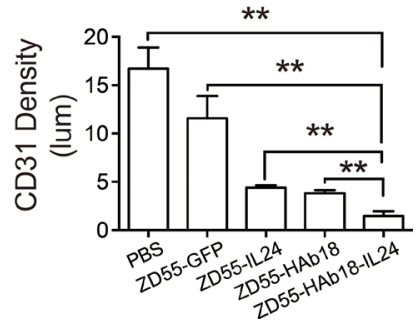

D

F
B
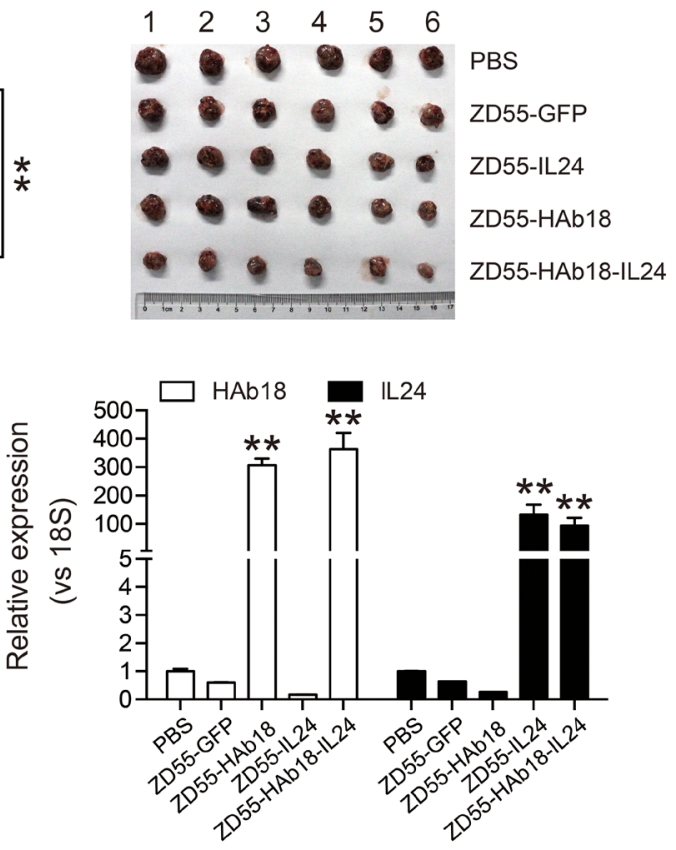

TUNEL Autophagosome CD31



Figure 5: ZD55-HAb18-IL24 displays antitumor activity in vivo. A. Growth curve of PLC/PRF/5 xenograft tumors after intratumoral injection of the indicated adenoviruses. Oncolytic adenoviruses were injected 13 days after subcutaneous inoculation of PLC/PRF/5 cells (day 0, arrow). The corresponding volumes of PBS were injected as a control. B, C. Tumors were excised, weighed, and photographed 33 days after injection. Significant decreases in tumor volume (B) and weight (C) were observed in the ZD55-HAb18-IL24treated group. The bars represent the mean \pm S.D. $(n=6)$. D. qRT-PCR detection of HAb18 and IL24 expression in tumors from each group. Data were normalized to 18S, and are shown as the fold change relative to PBS-treated cells. E. ELISA detection of HAb18 concentration in serum 33 days after virus injection. F. Representative images of TUNEL staining (scale bar, $100 \mu \mathrm{m}$; left panel), electron microscopy (scale bar, $200 \mathrm{~nm}$; middle panel), and CD31 immunohistochemical staining (scale bar, $100 \mu \mathrm{m}$; right panel) of tumor sections from each group. G. Quantification of the TUNEL staining results in (F). H. Quantification of CD31-positive cells in (F). The bars represent the mean \pm S.D. of triplicate samples. ${ }^{*} \mathrm{p}<0.05,{ }^{* *} \mathrm{p}<0.01$. 
Oncolytic adenoviral vectors can be generated using two strategies. One strategy is to use a tumorspecific promoter to drive an essential viral replication gene. Another strategy is to delete a viral element that is required for virus replication in normal cells but dispensable in tumor cells. The adenovirus E1B55KD enhances cyclin E expression and is required for efficient viral DNA replication [35]. E1B55KD deletion might attenuate oncolytic adenovirus replication in tumor cells. Luo et al. constructed the AdCN205-IL24 vector in which the hTERT promoter was used to control the expression of E1A in which the CR2 region was deleted. The expression of IL24 was controlled by the adenoviral E3 promoter. AdCN205-IL24 could express IL24 and suppress tumor growth [36].

Blocking HAb18G/CD147 using mAb HAb18 was shown to inhibit HCC growth in vivo [37]. Licartin $\left({ }^{131}\right.$ I-labled HAb18) was approved for clinical use by the Food and Drug Administration of China in April 2005 and is an effective and safe treatment for HCC [38]. IL24 has been widely investigated because it has selective antitumor effects in HCC [36, 39, 40]. Ad-IL24 was effective in phase I/II clinical trials in patients with advanced cancers [41]. We hypothesized that the combination of the HAb18 and IL24 could result in enhanced antitumor effects. We demonstrated that oncolytic adenoviruses carrying both genes more effectively inhibited HCC cell growth than either gene alone both in vitro and in vivo. Furthermore, increases in apoptosis and autophagy were observed in ZD55-HAb18-IL24-treated cells (Figure 4 and 5). Collectively, our data indicate that ZD55-HAb18-IL24 has anti-tumor effects and is a potential therapeutic strategy for HCC.

\section{MATERIALS AND METHODS}

\section{Patient samples}

Adjacent, non-cancerous tissue samples were anonymously obtained from $\mathrm{HCC}$ patients treated at the Center of Clinical Oncology at the Affiliated Hospital of Xuzhou Medical College. All human samples were obtained with informed consent, and approval for usage was obtained from the Ethics Committee of the Affiliated Hospital of Xuzhou Medical College. Studies using these samples were approved by the Institutional Review Board of the Institute of Biochemistry and Cell Biology, Shanghai Institutes for Biological Sciences, Chinese Academy of Sciences.

\section{Cell culture}

The human HCC cell lines (PLC/PRF/5, HepG2, BEL-7404, and Hep3B), human bladder cancer cell line (T24), human hepatocytes (QSG-7701), HEK-293T cells, and the murine colon cancer cell line (MC38) were purchased from the Cell Bank of the Type Culture Collection of the Chinese Academy of Sciences (Shanghai, China). The HEK-293 cell line was obtained from Microbix Biosystems Inc. (Toronto, Canada). All cells were cultured according to the manufacturer's instructions.

\section{Establishment of CD147-overexpressing and CD147-knockdown cells}

Full-length CD147 cDNA was amplified by PCR and cloned into the lentiviral vector pLVX. The lentiviral stock was produced in 293T cells by transfecting pLVX-CD147 and the lentivirus backbone plasmids pMD2G and pSPAX2. Cells were infected with the lentivirus vectors and selected with puromycin $(10 \mu \mathrm{g} / \mathrm{mL})$. For stable knockdown, shRNA against CD147 (5'-GGTTCTTCGTGAGTTCCTC-3', 5'-GTACAAGATCACTGACTCT-3') and a scramble control shRNA (5'-CTACCGTTGTTATAGGTG-3') were synthesized and cloned into the pLVX vector.

\section{Adenovirus construction}

The GFP, IL24, HAb18, and HAb18-F2A-IL24 expression cassettes were inserted into pShuttle-E1B $(\Delta 55)$ with E1B55K deletion. Oncolytic adenovirus plasmids were generated by homologous recombination of the shuttle vector and the adenoviral backbone plasmid in $E$. coli BJ5183 cells. Oncolytic adenoviruses were packaged and amplified in HEK-293 cells, and then purified by gradient $\mathrm{CsCl}$ centrifugation. Virus titer was measured using the Quick Titer Adenovirus Titer Immunoassay Kit (Cell Biolabs, San Diego, CA, USA), or plaque assays (HEK293 cells). Viral genomic DNA was extracted using the Blood Genome Extract Kit and the manufacturer's protocol (Generay, Shanghai, China) for identification. The absence of wild-type contamination was demonstrated by PCR with corresponding primers (Supplementary Table $\mathrm{S} 1)$. The F2A sequence was generated from the FMDV 2A sequence through the addition of a furin cleavage site sequence (RAKR) to the N-terminus of the FMDV 2A sequence.

\section{Quantitative RT-PCR}

Total RNA was isolated using TRIzol (CWBIO, Beijing, China). Single-strand cDNA was synthesized using the ReverTra Ace qPCR RT Kit (Toyobo, Osaka, Japan). RNA expression was analyzed using SuperReal Premix Plus (TIANGEN, Beijing, China) according to the manufacturer's protocols. The sequences of all primers are shown in Supplementary Table S1.

\section{Western blotting}

Protein concentrations were estimated using the Lowry assay (BioRad, Hercules, CA, USA). Western 
blotting was performed using standard protocols and the following antibodies: anti-procaspase-9, -procaspase-3, -PARP, -GFP (Santa Cruz biotechnology, Santa Cruz, CA, USA), -IL24 (GenHunter Corporation, Nashville, TN, USA), -HA, -actin, -GAPDH (CWBIO, Beijing, China), -LC3, -p62, and -beclin-1 (Sigma, St. Louis, MO, USA). All HRP-conjugated secondary antibodies were purchased from Santa Cruz Biotechnology.

\section{Proliferation assays}

Cell proliferation was measured using the CCK-8 kit (Dojindo, Kumamoto, Japan). Following treatment, the cells were incubated with $10 \mu \mathrm{L} \mathrm{CCK}-8$ at $37^{\circ} \mathrm{C}$ for $2 \mathrm{~h}$. Absorbance was measured at $450 \mathrm{~nm}$ and $630 \mathrm{~nm}$ using a Biotek Eon Microplate Reader.

\section{Migration assays}

Cell migration assays were performed using transwell inserts ( $8 \mu \mathrm{m}$, BD Biosciences, San Jose, CA, USA) placed in 24-well plates. Cells were cultured in the upper chambers of the transwell inserts in $200 \mu \mathrm{L}$ serum-free medium. The medium in the bottom chamber contained $10 \%$ fetal bovine serum. Following a $12 \mathrm{~h}$ incubation, the inserts were stained with $0.5 \%$ crystal violet and imaged.

\section{Flow cytometry}

Apoptosis was analyzed using the Annexin V-FITC Apoptosis Detection kit (Beyotime Biotechnology, Shanghai, China) according to the manufacturer's instructions. The mitochondrial membrane potential was evaluated by staining the cells with the JC-1 fluorescent probe (BD Biosciences) and then subjecting them to flow cytometry using a FACS Calibur flow cytometer (BD Biosciences).

\section{Immunofluorescence microscopy}

Cells were fixed with $4 \%(\mathrm{w} / \mathrm{v})$ paraformaldehyde, permeabilized using $0.1 \%(\mathrm{w} / \mathrm{v})$ Triton X-100 and blocked with $1 \%$ bovine serum albumin. The cells were stained with an anti-LC3 antibody (Sigma) overnight and then incubated with a Cy3-labeled secondary antibody in PBS with $1 \%$ FBS for 1 hour at room temperature. Images were captured using a laser scanning confocal microscope (Olympus, Tokyo, Japan).

\section{Animal experiments}

All in vivo experiments were performed according to protocols approved by the U.S. Public Health Service Policy on Humane Care and Use of Laboratory Animals. Four-week-old female BALB/c nude mice and C57/BL6 mice were purchased from the Animal Core Facility
(Shanghai, China). We pre-mixed $5 \times 10^{6} \mathrm{PLC} / \mathrm{PRF} / 5$ cells with Matrigel (BD Biosciences) at a 1:1 ratio and subcutaneously injected the cells into the right flank of each mouse. A total of $1 \times 10^{6}$ MC38 cells were injected into C57/BL6 mice. When the tumors reached 80-120 $\mathrm{mm}^{3}$, the mice were randomly divided into five groups (six mice per group). Oncolytic adenovirus $\left(2.5 \times 10^{8}\right.$ PFU per mouse) or PBS was intratumorally injected every other day (four injections total). The tumor volume was measured using a Vernier caliper every 4 days and calculated using the following equation: (length $\times$ width ${ }^{2}$ ) /2. At the end of the experiment, the tumors were resected from the sacrificed mice for immunohistochemical analysis. The levels of AST, ALT, creatinine, and BUN in serum were measured.

\section{ELISA}

Serum HAb18 expression was quantified by ELISA using a human IgG ELISA detection kit (Mlbio, Shanghai, China). All experiments were performed according to the manufacturer's protocols.

\section{Immunohistochemistry and TUNEL assays}

Tumor tissue was fixed in $4 \%$ formaldehyde overnight, embedded in paraffin, and sectioned $(5 \mu \mathrm{m}$ thickness) for immunohistochemical analysis. Sections were stained with an anti-CD31 antibody (Cell Signaling Technology, Danvers, MA, USA). TUNEL assays were performed according to the manufacturer's protocol (Roche, Basel, Switzerland). Hematoxylin was used as a counterstain.

\section{Transmission electron microscopy}

Tumor tissue was fixed with $2.5 \%$ glutaraldehyde overnight followed by $1 \%$ osmium tetroxide for $1.5 \mathrm{~h}$. The tissue samples were then dehydrated using a graded series of ethanol. Samples were then rinsed with acetone and permeated overnight with embedding buffer. Sections of $70 \mathrm{~nm}$ thickness were dual-stained with $2 \%$ uranyl acetate and lead citrate. Autophagosomes were examined by transmission electron microscopy (FEI, Hillsboro, OR, USA).

\section{Statistical analysis}

Data are presented as the mean \pm standard deviation (S.D). Comparisons between two groups were performed with Student's t-tests and the GraphPad Prism 6.0 software (GraphPad Software, USA).

\section{ACKNOWLEDGMENTS}

We thank Dr. Zhi-Nan Chen of the Fourth Military Medical University for providing the mAb HAb18 
construct. We thank Lan-Ying Sun for assistance with cell culture and the staff at the Cell Analysis Center and Animal Core Facility (Institute of Biochemistry and Cell Biology, Shanghai Institutes for Biological Sciences, Chinese Academy of Sciences) for providing technical assistance.

\section{CONFLICTS OF INTEREST}

The authors declare that there are no conflicts of interest.

\section{GRANT SUPPORT}

This work was supported by National Natural Science Fund (31301064, 81372453), Natural Science Foundation of Shanghai (13ZR1446300), Youth Innovation Promotion Association, Chinese Academy of Sciences (2013KIP101), and Shanghai Municipal Science and Technology Commission Fund (15431902800).

\section{REFERENCES}

1. Hanahan D, Weinberg Robert A. Hallmarks of Cancer: The Next Generation. Cell. 2011; 144:646-674.

2. Zhang YW, Niu J, Lu X, Yang YX, Zhao HW, He X, Yin GW, Wu JD, Yan DL, Sun JF, Wen JF, Feng JF, Xue HZ, Lau WY. Multi-target lentivirus specific to hepatocellular carcinoma: in vitro and in vivo studies. Journal of hepatology. 2013; 58:502-508.

3. Graepler F, Verbeek B, Graeter T, Smirnow I, Kong HL, Schuppan D, Bauer M, Vonthein R, Gregor M, Lauer UM. Combined endostatin/sFlt-1 antiangiogenic gene therapy is highly effective in a rat model of HCC. Hepatology. 2005; 41:879-886.

4. Ma L, Luo L, Qiao H, Dong X, Pan S, Jiang H, Krissansen GW, Sun X. Complete eradication of hepatocellular carcinomas by combined vasostatin gene therapy and B7H3-mediated immunotherapy. Journal of hepatology. 2007; 46:98-106.

5. Drozdzik M, Qian C, Xie X, Peng D, Bilbao R, Mazzolini G, Prieto J. Combined gene therapy with suicide gene and interleukin-12 is more efficient than therapy with one gene alone in a murine model of hepatocellular carcinoma. Journal of hepatology. 2000; 32:279-286.

6. Li Y, Xu J, Chen L, Zhong WD, Zhang Z, Mi L, Zhang Y, Liao CG, Bian HJ, Jiang JL, Yang XM, Li XY, Fan CM, Zhu P, Fu L, Chen ZN. HAb18G (CD147), a cancer-associated biomarker and its role in cancer detection. Histopathology. 2009; 54:677-687.

7. Marchiq I, Albrengues J, Granja S, Gaggioli C, Pouyssegur J, Simon MP. Knock out of the BASIGIN/CD147 chaperone of lactate/H+ symporters disproves its pro-tumour action via extracellular matrix metalloproteases (MMPs) induction.
Oncotarget. 2015; 6:24636-24648. doi: 10.18632/ oncotarget.4323.

8. Kuang YH, Chen X, Su J, Wu LS, Liao LQ, Li D, Chen ZS, Kanekura T. RNA interference targeting the CD147 induces apoptosis of multi-drug resistant cancer cells related to XIAP depletion. Cancer letters. 2009; 276:189-195.

9. Pan Y, He B, Song G, Bao Q, Tang Z, Tian F, Wang S. CD147 silencing via RNA interference reduces tumor cell invasion, metastasis and increases chemosensitivity in pancreatic cancer cells. Oncology reports. 2012; 27:2003-2009.

10. ZN C, YF L. Monoclonal antibody HAb18 to human hepatoma. Monoclonal Antibodies. 1990:8:11.

11. YF L, ZN C, YY J. Localization of hepatocellular carcinoma with monoclonal antibodies. Zhonghua Yi Xue Za Zhi. 1991; 71:362-365.

12. Toole BP. Emmprin (CD147), a cell surface regulator of matrix metalloproteinase production and function. Current topics in developmental biology. 2003; 54:371-389.

13. Xu J, Shen ZY, Chen XG, Zhang Q, Bian HJ, Zhu P, Xu HY, Song F, Yang XM, Mi L, Zhao QC, Tian R, Feng Q, et al. A randomized controlled trial of Licartin for preventing hepatoma recurrence after liver transplantation. Hepatology. 2007; 45:269-276.

14. Huang EY, Madireddi MT, Gopalkrishnan RV, Leszczyniecka M, Su Z, Lebedeva IV, Kang D, Jiang H, Lin JJ, Alexandre D, Chen Y, Vozhilla N, Mei MX, et al. Genomic structure, chromosomal localization and expression profile of a novel melanoma differentiation associated (mda-7) gene with cancer specific growth suppressing and apoptosis inducing properties. Oncogene. 2001; 20:7051-7063.

15. Nishikawa T, Ramesh R, Munshi A, Chada S, Meyn RE. Adenovirus-mediated mda-7 (IL24) gene therapy suppresses angiogenesis and sensitizes NSCLC xenograft tumors to radiation. Molecular therapy. 2004; 9:818-828.

16. Shi H, Wei LL, Yuan CF, Yang JX, Yi FP, Ma YP, Song FZ. Melanoma differentiation-associated gene-7/interleukin 24 inhibits invasion and migration of human cervical cancer cells in vitro. Saudi medical journal. 2007; 28:1671-1675.

17. Emdad L, Lebedeva IV, Su ZZ, Gupta P, Sarkar D, Settleman J, Fisher PB. Combinatorial treatment of nonsmall-cell lung cancers with gefitinib and Ad.mda-7 enhances apoptosis-induction and reverses resistance to a single therapy. Journal of cellular physiology. 2007; 210:549-559.

18. McKenzie T, Liu Y, Fanale M, Swisher SG, Chada S, Hunt KK. Combination therapy of Ad-mda7 and trastuzumab increases cell death in Her-2/neu-overexpressing breast cancer cells. Surgery. 2004; 136:437-442.

19. Toth K, Doronin K, Tollefson AE, Wold WS. A multitasking oncolytic adenovirus vector. Molecular therapy. 2003; 7:435-437. 
20. Dobbins GC, Ugai H, Curiel DT, Gillespie GY. A Multi Targeting Conditionally Replicating Adenovirus Displays Enhanced Oncolysis while Maintaining Expression of Immunotherapeutic Agents. PloS one. 2015; 10:e0145272.

21. Gomez-Gutierrez JG, Nitz J, Sharma R, Wechman SL, Riedinger E, Martinez-Jaramillo E, Sam Zhou H, McMasters KM. Combined therapy of oncolytic adenovirus and temozolomide enhances lung cancer virotherapy in vitro and in vivo. Virology. 2016; 487:249-259.

22. Kratzer F, Rosorius O, Heger P, Hirschmann N, Dobner T, Hauber J, Stauber RH. The adenovirus type 5 E1B-55K oncoprotein is a highly active shuttle protein and shuttling is independent of E4orf6, p53 and Mdm2. Oncogene. 2000; 19:850-857.

23. O'Shea CC, Johnson L, Bagus B, Choi S, Nicholas C, Shen A, Boyle L, Pandey K, Soria C, Kunich J, Shen Y, Habets G, Ginzinger D, McCormick F. Late viral RNA export, rather than $\mathrm{p} 53$ inactivation, determines ONYX-015 tumor selectivity. Cancer cell. 2004; 6:611-623.

24. Zhang ZL, Zou WG, Luo CX, Li BH, Wang JH, Sun LY, Qian QJ, Liu XY. An armed oncolytic adenovirus system, ZD55-gene, demonstrating potent antitumoral efficacy. Cell research. 2003; 13:481-489.

25. Zhao L, Gu J, Dong A, Zhang Y, Zhong L, He L, Wang Y, Zhang J, Zhang Z, Huiwang J, Qian Q, Qian C, Liu X. Potent antitumor activity of oncolytic adenovirus expressing mda-7/IL-24 for colorectal cancer. Human gene therapy. 2005; 16:845-858.

26. Pei Z, Chu L, Zou W, Zhang Z, Qiu S, Qi R, Gu J, Qian C, Liu X. An oncolytic adenoviral vector of Smac increases antitumor activity of TRAIL against HCC in human cells and in mice. Hepatology. 2004; 39:1371-1381.

27. Gou X, Ru Q, Zhang H, Chen Y, Li L, Yang H, Xing J, Chen Z. HAb18G/CD147 inhibits starvation-induced autophagy in human hepatoma cell SMMC7721 with an involvement of Beclin 1 down-regulation. Cancer science. 2009; 100:837-843.

28. Fang F, Wang L, Zhang S, Fang Q, Hao F, Sun Y, Zhao L, Chen S, Liao H, Wang L. CD147 modulates autophagy through the $\mathrm{PI} 3 \mathrm{~K} / \mathrm{Akt} / \mathrm{mTOR}$ pathway in human prostate cancer PC-3 cells. Oncology letters. 2015; 9:1439-1443.

29. Morisawa T, Marusawa H, Ueda Y, Iwai A, Okazaki IM, Honjo T, Chiba T. Organ-specific profiles of genetic changes in cancers caused by activation-induced cytidine deaminase expression. International journal of cancer. 2008; 123:2735-2740.

30. Tang J, Guo YS, Yu XL, Huang W, Zheng M, Zhou YH, Nan G, Wang JC, Yang HJ, Yu JM, Jiang JL, Chen ZN. CD147 reinforces $[\mathrm{Ca} 2+]$ i oscillations and promotes oncogenic progression in hepatocellular carcinoma. Oncotarget. 2015; 6:34831-34845. doi: 10.18632/oncotarget.5225.

31. Goldenberg DM, Cardillo TM, Govindan SV, Rossi EA, Sharkey RM. Trop-2 is a novel target for solid cancer therapy with sacituzumab govitecan (IMMU-132), an antibody-drug conjugate (ADC). Oncotarget. 2015; 6:22496-22512. doi: 10.18632/oncotarget.4318.

32. Armbruster C, Stiegler GM, Vcelar BA, Jager W, Michael NL, Vetter N, Katinger HW. A phase I trial with two human monoclonal antibodies (hMAb 2F5, 2G12) against HIV-1. Aids. 2002; 16:227-233.

33. Lewis AD, Chen R, Montefiori DC, Johnson PR, Clark KR. Generation of neutralizing activity against human immunodeficiency virus type 1 in serum by antibody gene transfer. Journal of virology. 2002; 76:8769-8775.

34. Wei D, Li Q, Wang XL, Wang Y, Xu J, Feng F, Nan G, Wang B, Li C, Guo T, Chen ZN, Bian H. Oncolytic Newcastle disease virus expressing chimeric antibody enhanced antitumor efficacy in orthotopic hepatoma-bearing mice. J Exp Clin Cancer Res. 2015; 34:153.

35. Zheng X, Rao XM, Gomez-Gutierrez JG, Hao H, McMasters KM, Zhou HS. Adenovirus E1B55K Region Is Required To Enhance Cyclin E Expression for Efficient Viral DNA Replication. Journal of virology. 2008; 82:3415-3427.

36. Luo J, Xia Q, Zhang R, Lv C, Zhang W, Wang Y, Cui Q, Liu L, Cai R, Qian C. Treatment of cancer with a novel dualtargeted conditionally replicative adenovirus armed with mda-7/IL-24 gene. Clin Cancer Res. 2008; 14:2450-2457.

37. Xu J, Xu HY, Zhang Q, Song F, Jiang JL, Yang XM, Mi L, Wen N, Tian R, Wang L, Yao H, Feng Q, Zhang Y, Xing JL, Zhu P, Chen ZN. HAb18G/CD147 functions in invasion and metastasis of hepatocellular carcinoma. MCR. 2007; 5:605-614.

38. Chen ZN, Mi L, Xu J, Song F, Zhang Q, Zhang Z, Xing JL, Bian HJ, Jiang JL, Wang XH, Shang P, Qian AR, Zhang $\mathrm{SH}$, et al. Targeting radioimmunotherapy of hepatocellular carcinoma with iodine (131I) metuximab injection: clinical phase I/II trials. International journal of radiation oncology, biology, physics. 2006; 65:435-444.

39. Li QY, Shi Y, Huang DH, Yang T, Wang JH, Yan GH, Wang HY, Tang XJ, Xiao CY, Zhang WJ, Zhang M, Wang L, Gong Y, Yang W, Wu XY, Xiang Y. Cytokine-induced killer cells combined with dendritic cells inhibited liver cancer cells. International journal of clinical and experimental medicine. 2015; 8:5601-5610.

40. Chen WY, Cheng YT, Lei HY, Chang CP, Wang CW, Chang MS. IL-24 inhibits the growth of hepatoma cells in vivo. Genes and immunity. 2005; 6:493-499.

41. Menezes ME, Bhatia S, Bhoopathi P, Das SK, Emdad L, Dasgupta S, Dent P, Wang XY, Sarkar D, Fisher PB. MDA-7/IL-24: multifunctional cancer killing cytokine. Advances in experimental medicine and biology. 2014; 818:127-153. 